\title{
Evaluation of Airway Volume Changes in Patients Treated with Distraction Osteogenesis: A Pilot Study
}

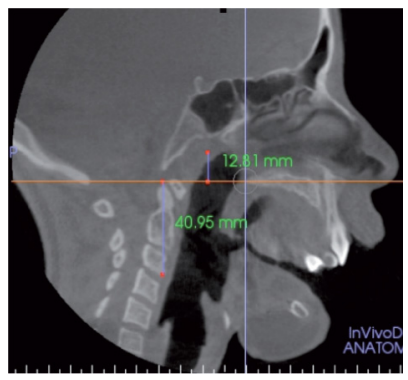

\author{
Manish VALIATHAN, Tasha METZGERA, Hakan EL, \\ Mark G. HANS, Martin J PALOMO
}

\section{ABSTRACT}

The use of distraction osteogenesis to improve the airway volume of individuals affected by craniofacial anomalies is common practice today. The methods of assessing the outcomes of such surgical procedures have changed over the last several years.

Objective : The objective of the present pilot study was to determine if the CBCT imaging modality may serve as a simple and reliable method for craniofacial practitioners to evaluate airway volume changes following distraction osteogenesis.

Materials and Methods : Twelve patients who had previously undergone distraction osteogenesis for the primary purpose of improving their airways were enrolled in the current study. Pre (TO) and post-surgical (T1) CBCT's volumes were analyzed to measure the nasopharyngeal (NP) and oropharyngeal (OP) volume changes. InVivoDental 4.0 (Anatomage Inc., San Jose, CA) (IVD) program was used to visualize and render the oropharyngeal (OP) and nasal passage (NP) volumes, separately. Means and standard deviations were calculated.

Results: Of the 7 males and 5 females in the study, 4 patients underwent mandibular distraction (MandDO) and 8 underwent maxillary distraction (MaxDO). Four in the MaxDO group were treated with internal distractors and 4 were treated with external distractors. Individuals who underwent internal 
MaxDO greatly improved their NP volumes (1605 \pm 1736 to $3273 \pm 3130 \mathrm{~mm}^{3}$ ) as did patients who underwent external MaxDO (5763 \pm 5077 to $\left.9243 \pm 9442 \mathrm{~mm}^{3}\right)$. In comparison, the MandDO group's NP volume showed modest gains (3519 \pm 1944 to $\left.3894 \pm 2516 \mathrm{~mm}^{3}\right)$. With regard to the $O P$ volumes, the MandDO group gained substantially (4906 \pm 2347 to $11385.8 \pm$ $6393.5 \mathrm{~mm}^{3}$ ) while the MaxDO groups showed humble increases; internal MaxDO group (1779 \pm 273.8 to $\left.2639.5 \pm 898.4 \mathrm{~mm}^{3}\right)$ and the external MaxDO group $(8959 \pm 5311$ to $9734.13 \pm 7124.7$ $\mathrm{mm}^{3}$ ).

Conclusions : In the present preliminary study of assessing airway volume changes using CBCT on patients who have undergone different DO techniques, MandDO greatly increases $O P$ volumes and MaxDO tends to increase NP volumes. The CBCT imaging modality holds great promise for craniofacial practitioners in its application to assess airways of individuals affected by craniofacial anomalies.

\title{
KEYWORDS
}

\author{
Airway volume \\ Distraction osteogenesis \\ CBCT imaging.
}

\section{INTRODUCTION}

Craniofacial anomalies involving the midface, such as craniofacial synostoses (Crouzon, Apert and Pfeiffer syndromes), anomalies involving primarily the mandible (Nager, Sticklers and Pierre Robin sequence) or anomalies associated with both the maxilla and mandible (Treacher Collins syndrome, hemifacial microsomia) can lead to a decrease in volume of the oropharyngeal and nasopharyngeal airway. The altered size and position of the maxilla and mandible typically cause a retro displacement of the tongue which results in a reduction of the oropharyngeal airway and may lead to upper airway obstruction. Affected patients often suffer from Obstructive Sleep Apnea (OSA) and in severe cases are treated by early tracheal intubation and tracheostomies ${ }^{1}$.

Formerly, a variety of surgical procedures were indicated for these patients in order to increase airway volume or allow for easier respiration. Distraction Osteogenesis (DO) has become an accepted method of treatment for patients requiring reconstruction of hypoplastic mandibles/maxillae and achieve lengthening of the jaw without the need for bone grafts ${ }^{1}$. DO is based on manipulation of a healing bone and stretching an osteotomized area before calcification has occurred, 
to generate additional bone and investing soft tissue ${ }^{2}$. Such procedures aim to lengthen, modify and reposition the affected mandible/maxilla by performing one or more osteotomies, manipulating the resulting bone fragments into a more desirable configuration, and "growing" the mandible through daily distraction ${ }^{3}$. The goal of the procedure is to reposition the bones in a more anterior position through the process of distraction and thereby open up the airway.

The advent of cone-beam computerized tomography (CBCT) in this decade, allows us to quantitatively evaluate the cross-sectional area and the 3-dimensional volume of the airway utilizing a lower radiation levels than traditional medical CT's, with a rapid ( $<20$ seconds) scan that is noninvasive ${ }^{4}$.

The information within the (3D) CBCT image data includes hard and soft tissue structures, allowing visualization of the pharyngeal airway without any obstruction of bony structures. $^{5}$ Segmentation of the nasopharyngeal and oropharyngeal airways, allows for these compartments to be independently evaluated.

The purpose of this retrospective, descriptive, pilot study was to use CBCT images of patients affected by craniofacial anomalies, who had undergone DO, and to compare the presurgical to post-surgical changes in

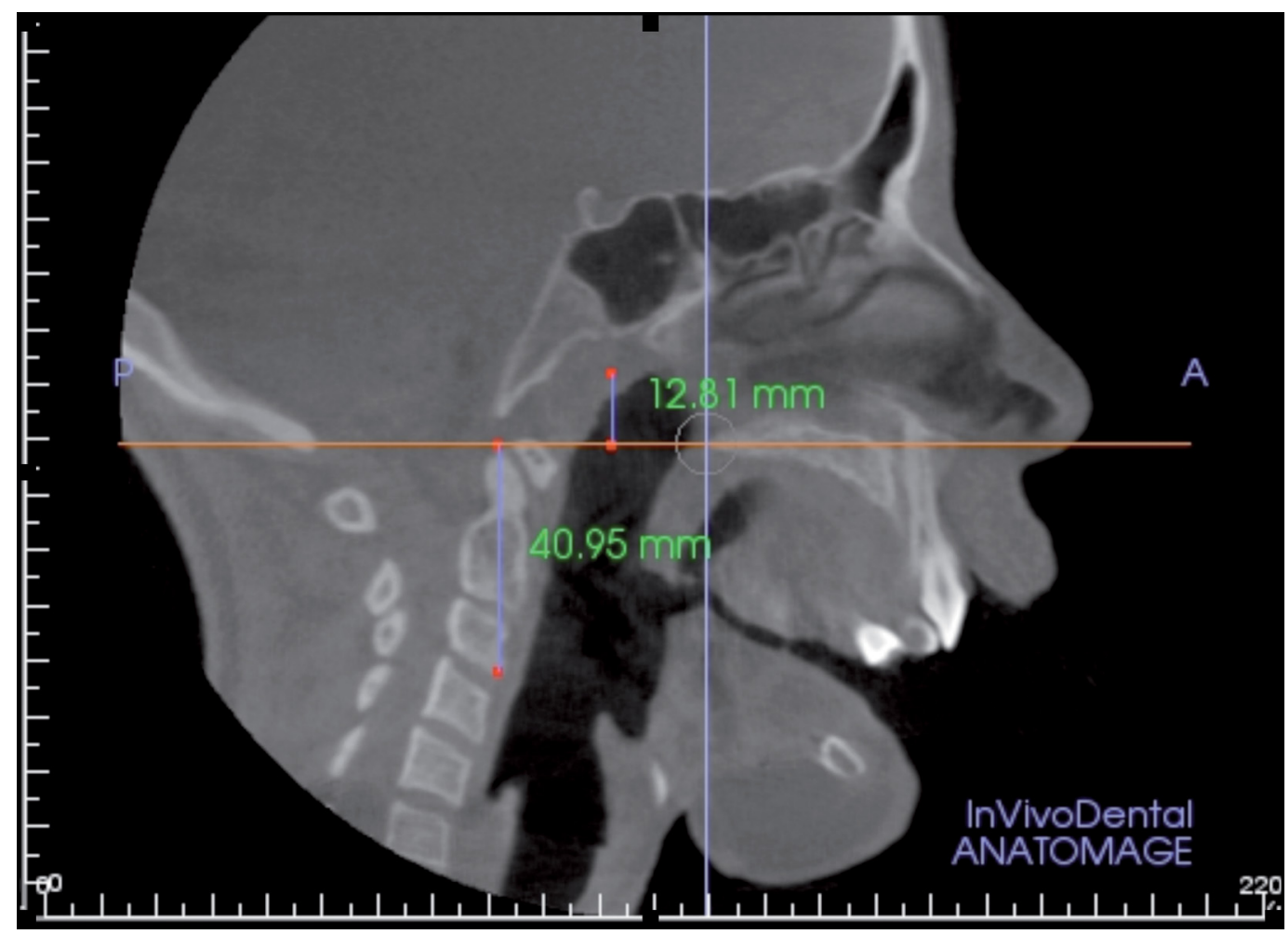

Figure 1

Illustrates the superior and inferior borders of the Nasopharyngeal (NP) and oropharyngeal (OP) volumes respectively. 


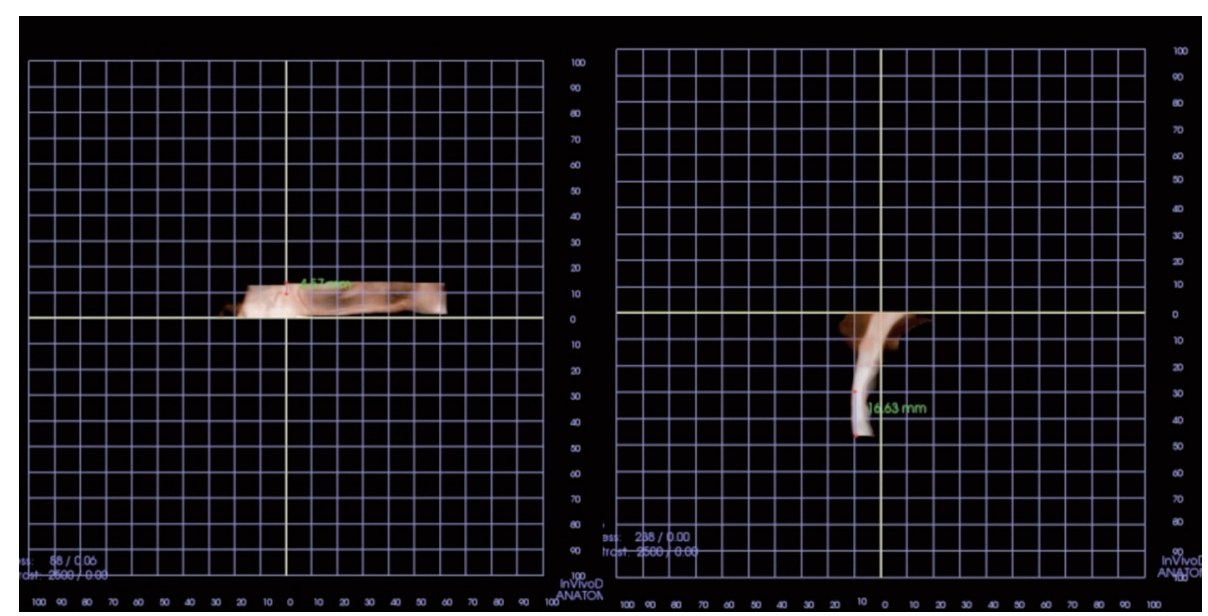

Figures 2 and 3

Segmented NP and OP volumes.

nasopharyngeal (NP) and oropharyngeal (OP) airway volumes. Specifically, we intended to identify if the CBCT imaging modality could detect changes in the airway of patients treated with DO in this pilot study.

\section{MATERIALS AND METHODS}

The experimental protocol used in this study was approved by the Case Western Reserve University Institutional Review Board. Seventeen patients affected by different craniofacial anomalies and previously treated with DO were selected from the pool of patients in the Department of Orthodontics. Due to extensive previous histories of DO, unavailability of pre or post operative CBCT scans, and/or unidentifiable landmarks, five patients were excluded from the study. The final sample consisted of 12 individuals ( 7 males and 5 females) and ranged in age from 3 years 8 months to 17 years 2 months. All included patients had initial (TO) and postoperative (T1) CBCT scans. Of the 12 patients, 8 were treated with maxillary distraction (MaxDO) and four with mandibular distraction (MandDO). 4 of the MaxDO patients were treated with internal distractors and 4 with external distractors. In order to blind the operator, subject volumes were de-identified and the volume sets were not separated based on the procedure (MaxDO or MandDO). Similar DO protocol was used for the subjects. Distraction commenced at a rate of $1 \mathrm{~mm} /$ day after a 5-7 day latency period. Duration of distraction was based on the improvement of the maxillary/mandibular position and improvement in airway. Anterior crossbite/overjet and was recorded clinically. In most cases, intentional overcorrection was achieved to negate a tendency to relapse and to account for continued growth. 
The InVivoDental 4.0 (Anatomage Inc., San Jose, CA) (IVD) program was used to visualize and render the oropharyngeal (OP) and nasal passage (NP) volumes, separately. Patients were first viewed in the sagittal plane. In order to find the midsagittal point, images were scanned until the incisal foramen was readily visible as well as the anterior nasal spine and posterior nasal spine. Once this position was identified, images were reoriented so that their horizontal plane aligned through the anterior nasal spine and posterior nasal spine. OP volume was defined as the volume of pharynx between the palatal plane (ANS-PNS) extending to the posterior wall of the pharynx and the plane that is parallel to the palatal plane which passes from the most anteroinferior point of the third cervical vertebrae (Fig. 1). The inferior limit of the NP airway was defined as the superior limit of the OP airway and the superior limit was defined as the last slice before the nasal septum fused with the posterior wall of the pharynx. In order to better view this fusion, the superior border of the NP was defined on the axial slice first and then reflected to the sagittal plane. The NP volume was rendered as a whole structure and included nasopharynx, turbinates and nares. The described airway volumes were rendered with the IVD program, according to the manufacturer's recommendations ${ }^{6}$. The IVD program allows the user to "sculpt out" the desired airway volume from the rest of the 3D structure, and by adjusting the brightness and opacity values one can clean out the unwanted voxels before calculating the final airway volume (Fig. 2 and 3). The program also allows the user change the threshold values in order to obtain a solid airway volume. Operator reliability was tested by repeating all measurements 2 weeks apart and using the Spearman's rho correlation coefficient. The pre-distraction and postdistraction Spearman's $=0.965$ and 0.984 respectively. Both were deemed highly significant.

\section{RESULTS}

The descriptive demographics of our data are provided in Table I. The mean age of the total sample in the beginning of treatment (TO) was $10.28 \pm 5.27$ years

Age at T0 ranged from 3.67 years to 20.83 years. Mean age at the postoperative stage (T1) was $12.12 \pm 5.34$ years and ranged from 4.08 years to 21.25 years.

Table II and III present the individual cases with the means \pm standard deviations grouped by the type of DO performed.

Of the 7 males and 5 females in the study, 4 patients underwent mandibular distraction (MandDO) and 8 underwent maxillary distraction (MaxDO). 4 in the MaxDO group were treated with internal distractors and 4 were treated with external distractors.

\footnotetext{
The most prominent change in OP airway volume was observed for
} 


\begin{tabular}{|c|c|c|c|c|}
\hline Type of DO & Gender & $\begin{array}{l}\text { Age at Initial } \\
\text { Examination }\end{array}$ & Days from T0-T1 & $\begin{array}{l}\text { Mean \# of days } \\
\text { from TO-T1 }\end{array}$ \\
\hline \multirow{4}{*}{$\begin{array}{l}\text { Mandibular } \\
\text { Distraction } \\
\text { Patients }\end{array}$} & M & 11 Years, 0 Months & 855 & \multirow{4}{*}{$958.25 \pm 157$} \\
\hline & $\mathrm{M}$ & 4 Years, 6 Months & 911 & \\
\hline & $\mathrm{F}$ & 11 Years, 8 Months & 875 & \\
\hline & $\mathrm{F}$ & 11 Years, 6 Months & 1192 & \\
\hline \multirow{4}{*}{$\begin{array}{c}\text { Maxillary } \\
\text { Distraction } \\
\text { Patients } \\
\text { (Internal Distractor) }\end{array}$} & M & 13 Years, 10 Months & 372 & \multirow{4}{*}{$344.75 \pm 218$} \\
\hline & $\mathrm{F}$ & 3 Years, 8 Months & 144 & \\
\hline & $\mathrm{F}$ & 8 Years, 10 Months & 641 & \\
\hline & $\mathrm{M}$ & 4 Years, 0 Months & 222 & \\
\hline \multirow{4}{*}{$\begin{array}{c}\text { Maxillary } \\
\text { Distraction } \\
\text { Patients (External } \\
\text { Distractor) }\end{array}$} & M & 9 Years, 2 Months & 503 & \multirow{4}{*}{$458.25 \pm 291$} \\
\hline & $\mathrm{F}$ & 7 Years, 2 Months & 841 & \\
\hline & $M$ & 20 Years, 10 Months & 160 & \\
\hline & $\mathrm{M}$ & 17 Years, 2 Months & 329 & \\
\hline
\end{tabular}

Table 1:

Demographics and procedures performed on all 12 patients with time points in days from TO-T1.

\begin{tabular}{|c|c|c|c|c|c|}
\hline Type of DO & Patient ID & Gender & $\begin{array}{l}\text { NP volume } \\
\text { T0 }\left(\mathrm{mm}^{3}\right)\end{array}$ & $\begin{array}{l}\text { NP volume } \\
\text { T1 }\left(\mathrm{mm}^{3}\right)\end{array}$ & NP\% Change \\
\hline \multirow{4}{*}{ MandDO } & Case ID 1 & M & 1316 & 1045 & -20.59 \\
\hline & Case ID 2 & $M$ & 4994 & 4827 & -3.34 \\
\hline & Case ID 3 & $\mathrm{~F}$ & 4441 & 4712 & 6.10 \\
\hline & Case ID 4 & $\mathrm{~F}$ & 4246 & 5811 & 36.86 \\
\hline Means \pm stdev & & & $3519 \pm 1944$ & $3894 \pm 2516$ & \\
\hline \multirow{4}{*}{$\begin{array}{c}\text { MaxDO } \\
\text { (Internal } \\
\text { Distractor) }\end{array}$} & Case ID 5 & M & 3744 & 6423 & 71.55 \\
\hline & Case ID 6 & $\mathrm{~F}$ & 153 & 288 & 88.24 \\
\hline & Case ID 7 & $\mathrm{~F}$ & 2289 & 5487 & 139.71 \\
\hline & Case ID 8 & $\mathrm{M}$ & 232 & 892 & 284.48 \\
\hline Means \pm stdev & & & $1605 \pm 1736$ & $3273 \pm 3130$ & \\
\hline \multirow{4}{*}{$\begin{array}{c}\text { MaxDO } \\
\text { (External } \\
\text { Distractor) }\end{array}$} & Case ID 9 & M & 2215 & 2606 & 17.65 \\
\hline & Case ID 10 & $\mathrm{~F}$ & 678 & 1307 & 92.77 \\
\hline & Case ID 11 & $\mathrm{M}$ & 10980 & 21737 & 97.97 \\
\hline & Case ID 12 & $M$ & 9170 & 11323 & 23.36 \\
\hline Means \pm stdev & & & $5763 \pm 5077$ & $9243 \pm 9442$ & \\
\hline
\end{tabular}

Table II:

NP volume changes denoted in means and standard deviations, from TO to T1, for the three different distractors used. 


\begin{tabular}{|c|c|c|c|c|c|}
\hline Type of DO & Patient & Gender & $\begin{array}{l}\text { OP volume } \\
\text { T0 }\left(\mathrm{mm}^{3}\right)\end{array}$ & $\begin{array}{l}\text { OP volume } \\
\text { T1 }\left(\mathrm{mm}^{3}\right)\end{array}$ & OP\% Change \\
\hline \multirow{4}{*}{ MandDO } & Case ID 1 & M & 5783 & 7924.50 & 37.03 \\
\hline & Case ID 2 & $M$ & 2246 & 4760.00 & 111.93 \\
\hline & Case ID 3 & $\mathrm{~F}$ & 2906.5 & 19246.50 & 562.19 \\
\hline & Case ID 4 & $\mathrm{~F}$ & 6688 & 13612.50 & 103.54 \\
\hline Means \pm stdev & & & $4906 \pm 2347$ & $11385.88 \pm 6393.5$ & \\
\hline \multirow{4}{*}{$\begin{array}{c}\text { MaxDO } \\
\text { (internal } \\
\text { Distractor) }\end{array}$} & Case ID 5 & M & 1962 & 2520.50 & 28.47 \\
\hline & Case ID 6 & $\mathrm{~F}$ & 1388 & 3760.50 & 170.93 \\
\hline & Case ID 7 & $\mathrm{~F}$ & 1790 & 2708.00 & 51.83 \\
\hline & Case ID 8 & $\mathrm{M}$ & 1975 & 1569.00 & -20.56 \\
\hline Means \pm stdev & & & $1779 \pm 273.8$ & $2639.5 \pm 898.4$ & \\
\hline \multirow{4}{*}{$\begin{array}{c}\text { MaxDO } \\
\text { (internal } \\
\text { Distractor) }\end{array}$} & Case ID 9 & M & 3736 & 1966.00 & -47.38 \\
\hline & Case ID 10 & $\mathrm{~F}$ & 5320 & 5535.50 & 4.06 \\
\hline & Case ID 11 & M & 14944 & 16882.00 & 12.97 \\
\hline & Case ID 12 & $\mathrm{M}$ & 11837 & 14553.00 & 22.95 \\
\hline Means \pm stdev & & & $8959 \pm 5311$ & $9734.13 \pm 7124.7$ & \\
\hline
\end{tabular}

Table III:

OP volume changes denoted in means and standard deviations, from TO to T1, for the three different distractors used.

MandDO patients. The volume difference between T0 and T1 time intervals in MandDO subjects $(4906 \pm 2347$ to $11385.8 \pm 6393.5 \mathrm{~mm}^{3}$ ) was substantially greater than the changes noted in the internal $(1779 \pm 273.8$ to $2639.5 \pm 898.4 \mathrm{~mm}^{3}$ ) and external $(8959 \pm 5311$ to $9734.13 \pm$ $7124.7 \mathrm{~mm}^{3}$ ) MaxDO subjects.

\section{DISCUSSION}

Recent advances in CBCT technology makes it a popular static imaging modality to evaluate the airway. The reason for this interest is mainly due to the considerably lower radiation
On the contrary, Individuals who underwent internal MaxDO greatly improved their NP volumes $\left(1605 \pm 1736\right.$ to $\left.3273 \pm 3130 \mathrm{~mm}^{3}\right)$ as did patients who underwent external MaxDO (5763 \pm 5077 to $\left.9243 \pm 9442 \mathrm{~mm}^{3}\right)$. In comparison, the MandDO group's NP volume showed modest gains (3519 \pm 1944 to $3894 \pm 2516 \mathrm{~mm}^{3}$ ).

doses of CBCT devices when compared to helical CTs ${ }^{7,8}$.

There is also a significant improvement in soft tissue contrast when compared to X-ray cephalometry ${ }^{9}$. 


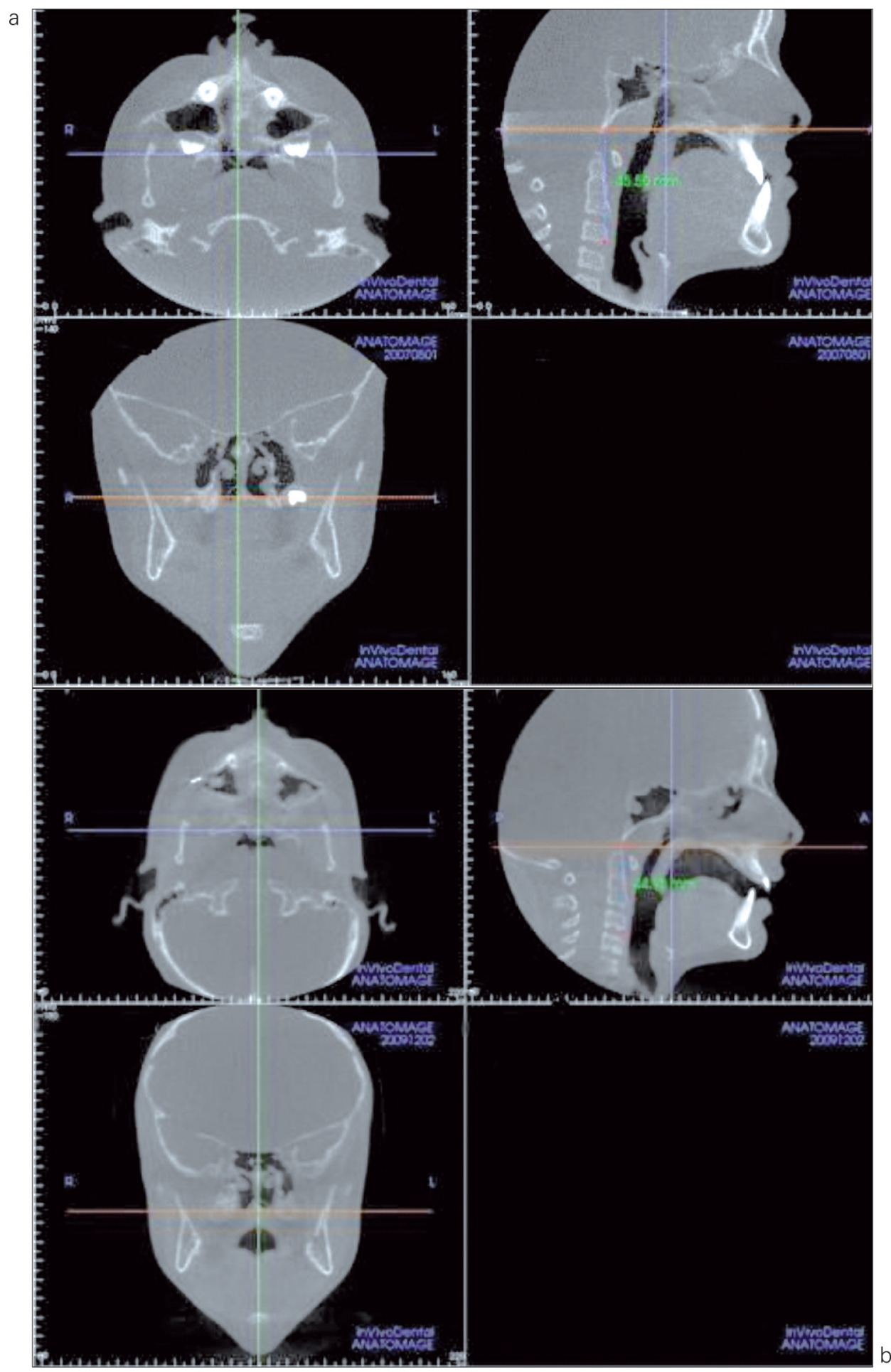

Figures 4 and 5

Noticeable pre to post distraction changes on an individual who underwent external distraction of the nasomaxillary complex. 
The use of commercially available or manufacturer supplied segmentation programs allows precise measurements of cross-sectional areas at different levels as well as threedimensional reconstruction and volumetric assessment. In a previous study, the intraoperator reliability of the IVD program, an automatic segmentation program, was found high ${ }^{10}$. The same methodology was used as in the previous study and one calibrated operator (T.E.M.) rendered and measured all of the airway volumes using the same software package. Therefore, when proper technique and protocol are followed, the validity of this method may be considered high. Most of the patients with craniofacial anomalies require airway management ranging from prone positioning to tracheotomy and $\mathrm{DO}^{11}$. DO is accepted as a reliable surgical technique and an alternative to tracheotomy for long-term management of the airway ${ }^{11,12,13}$. It has been observed that DO reduces the need for tracheotomy especially in pediatric patients and allows for early decannulation in patients who previously underwent tracheotomy ${ }^{14,15}$. In addition, oral feeding is significantly improved after $\mathrm{DO}^{16,17}$. Surgical intervention using DO in early childhood is a common approach. However, children with less obvious craniofacial anomalies and mild airway competence problems may seek treatment later in adolescence, even in their adult years. ${ }^{18}$ DO treatment is also performed successfully in the treatment of patients with craniofacial anomalies during their adolescent and adult years as noted in our sample.

Different types of distraction devices are used for the treatment of craniofacial anomalies. These are mainly classified as external and internal distraction devices and they have been used extensively for the maxillary and mandibular region according to the patients' needs. In the current study, subjects with mandibular retrusion were treated with internal and external mandibular distraction devices. However, given the small sample and the limited objective of the study; to evaluate airway changes using the CBCT modality, no effort was made to create sub-groups. Subjects with maxillary hypoplasia were treated with external and internal devices. Internal devices require a second surgery for removal and a greater degree of difficulty has been associated with controlling the distraction vector ${ }^{19}$. In order to eliminate the need for surgical removal at the end of treatment, several resorbable distraction devices have also been introduced more recently ${ }^{20,21}$. Patients tend to tolerate the internal devices better. Perhaps the main disadvantages of external distraction devices are their bulky construction and the psychosocial impact on the patients' life $^{22}$. Yet, they appear to provide more extended bone osteogenesis advancement when compared to internal devices and the distraction vectors are under better operator control $^{23,24,25}$. This may explain why a bigger NP airway volume change has been obtained in external MaxDO subjects when compared to the internal MandDO subjects (Fig. 4 and 5). It was an interesting finding that the changes mainly occurred in the NP region in the MaxDO patients and in the OP region for the MandDO patients. Xu et al. conducted a CT study on 11 patients with severe 
midface retrusion in young patients who underwent a LeFort III osteotomy with DO and concluded that DO can significantly improve the upper airway space $^{26}$. However, they noted that the enlargement in airway space was especially above the uvula and posterior nasal spine level (nasal cavity region) which was in agreement with the current study. They were unable to show any statistically significant difference for the airway volume at the level of epiglottis and esophagus. On the other hand, hyoid bone is known to have an important effect over the upper airway volume, especially the OP region. When the anatomic relationship of the hyoid bone to the soft tissues of the mandible and upper airway are taken into account, any displacement of the hyoid bone away from the posterior pharyngeal wall is believed to aid in increasing the airway size 27,28 . Furthermore, the small size of the mandible and its retruded position, cause a corresponding retrodisplacement of the tongue and reduction in the OP airway. In such conditions mandibular DO has been proposed as a useful method to resolve OP airway obstruction 29,30,31. The mean OP volume at T1 was aproximately 2.5 times the TO time

\section{CONCLUSIONS}

The current study indicates that distraction osteogenesis enhances the airway and is an extremely useful surgical technique in managing the airway of individuals affected by interval. This finding supports the aforementioned studies in terms of effectiveness of mandibular DO treatment over the OP airway.

There were limitations to the study that must be addressed.

- The limited sample size does not allow us to make definite conclusions.

- We did not have a homogeneous sample in terms of age/ gender/specific anomaly. However, we feel comfortable demonstrating, as many others referenced from the past, that DO is a valuable surgical technique in improving airway volume. Importantly, to our knowledge, this is the first CBCT study, albeit a pilot, that demonstrates the use of CBCT technology to effectively evaluate the effects of DO on NP and OP airway volume.

- The average number of days that elapsed before the T1 CBCT volumes may also come in to question. However, this is not an issue easily controlled as the resolution of post surgical edema shows large individual variations.

Further studies are needed to validate trends identified in this pilot effort.

craniofacial anomalies. Additionally, the study suggests that CBCT imaging modality is a beneficial and reliable method of assessing the changes in the airway. 


\section{REFERENCES}

1. Rachmiel, A., Aizenbud, D., Pillar, G., Srouji, S., \& Peled, M. Bilateral mandibular distraction for patients with compromised airway analyzed by three-dimensional CT. Int J Oral and Maxillofac Surg. 2005, 34(1); 9-18.

2. Proffit, W. R., Fields, H. W., \& Sarver, D. M. (2007) Contemporary Orthodontics (4th ed.). St. Louis: Mosby Elsevier.

3. Meehan, M., Maurer, C., Rohlfing, T., Shahidi, R., Rao, A., \& Girod, S. Virtual 3D planning and guidance of mandibular distraction osteogenesis. Comput Aided Surg. 2006, 11(2); 51-62.

4. Tso, H., Lee, J., Huang, J., Maki, K., Hatcher, D., \& Miller, A. (2009). Evaluation of the human airway using cone-beam computerized tomography. Oral Surg Oral Med Oral Pathol Oral Radiol Endod. 2009, 108(5); 768-76.

5. Kagan, D., Ueki, K., Hashiba, Y., Marukawa, K., Nakagawa, K., \& Yamamoto, E. (2008). A comparative CT evaluation of pharyngeal airway changes in class III patients receiving bimaxillary surgery or mandibular setback surgery. Oral Surg Oral Med Oral Pathol Oral Radiol Endod. 2008, 105(4); 495-502.

6. InVivoDental 4.0 Reference Manual. San Jose, CA: Anatomage Inc.; 2006.

7. Scarfe WC, Farman AG, Sukovic P. Clinical applications of cone-beam computed tomography in dental practice. J Can Dent Assoc. 2006, 72; 75-80.

8. Palomo JM, Rao PS, Hans MG. Influence of CBCT exposure conditions on radiation dose. Oral Surg Oral Med Oral Pathol Oral Radiol Endod. 2008, 105; 773-782.

9. Stuck BA, Maurer JT. Airway evaluation in obstructive sleep apnea. Sleep Med Rev 2008, 12; 411-436.

10. El H, Palomo JM. Measuring the airway in 3 dimensions: A reliability and accuracy study.: Accepted for publication; Am J Orthod Dentofac Orthop. 2010.

11. Perkins JA, Sie KCY, Milczuk H, et al.: Airway management in children with craniofacial anomalies. Cleft Palate Craniofac J 1997, 34:135-140.

12. Sculerati N, Gottlieb MD, Zimbler MS, et al.: Airway management in children with major craniofacial anomalies. Laryngoscope 1998, 108:1806-1812.

13. Boston M, Rutter MJ. Current airway management in craniofacial anomalies. Curr Opin Otolaryngol Head Neck Surg 2003;11:428-432.

14. Genecov DG, Barcelo CR, Steinberg D, Trone T, Sperry E. Clinical experience with the application of distraction osteogenesis for airway obstruction. J Craniofac Surg 2009;20 Suppl 2:1817-1821.,

15. Senders CW, Kolstad CK, Tollefson TT, Sykes JM. Mandibular distraction osteogenesis used to treat upper airway obstruction. Arch Facial Plast Surg 2010;12:11-15.

16. Looby JF, Schendel SA, Lorenz HP, Hopkins EM, Aizenbud D. Airway analysis: with bilateral distraction of the infant mandible. J Craniofac Surg 2009;20:1341-1346.

17. Monasterio FO, Molina F, Berlanga F, et al. Swallowing disorders in Pierre Robin sequence: its correction by distraction. J Craniofac Surg 2004;15:934-941.

18. Boston M, Rutter MJ. Current airway management in craniofacial anomalies. Curr Opin Otolaryngol Head Neck Surg 2003;11:428-432.

19. Pelo S, Gasparini G, Di Petrillo A, Tamburrini G, Di Rocco C. Distraction osteogenesis in the surgical treatment of craniostenosis: a comparison of internal and external craniofacial distractor devices. Childs Nerv Syst 2007;23:1447-1453.

20. Cohen SR, Holmes RE. Internal Le Fort III distraction with biodegradable devices. J Craniofac Surg 2001;12:264-72.

21. Burstein FD, Williams JK, Hudgins R, Graham L, Teague G, Paschal M, et al. Singlestage craniofacial distraction using resorbable devices. J Craniofac Surg 2002;13:77682.

22. Pelo S, Gasparini G, Di Petrillo A, Tamburrini G, Di Rocco C. Distraction osteogenesis in the surgical treatment of craniostenosis: a comparison of internal and external craniofacial distractor devices. Childs Nerv Syst 2007;23:1447-1453. 
23. Gosain AK, Santoro TD, Havlik RJ, Cohen SR, Holmes RE (2002) Midface distraction following Le Fort III and monobloc osteotomies: problems and solutions. Plast Reconstr Surg 109(6):1797-1808.

24. Kubler AC, Speder B, Zoller JE. (2004). Fronto-orbital advancement with simultaneous LeFort III-distraction. Craniomaxillofac Surg 32(5):291-295.

25. Pelo S, Gasparini G, Di Petrillo A, Tamburrini G, Di Rocco C. Distraction osteogenesis in the surgical treatment of craniostenosis: a comparison of internal and external craniofacial distractor devices. Childs Nerv Syst 2007;23:1447-1453.

26. $\mathrm{Xu} \mathrm{H}, \mathrm{YuZ}, \mathrm{MuX}$. The assessment of midface distraction osteogenesis in treatment of upper airway obstruction. J Craniofac Surg 2009;20 Suppl 2:1876-1881.

27. Williams, J. K., Maull, D., Grayson, B. H., Longaker, M. T., and McCarthy, J. G. Early decannulation with bilateral mandibular distraction for tracheostomy-dependent patients. Plast. Reconstr. Surg. 103: 48, 1999.

28. Perlyn CA, Schmelzer RE, Sutera SP, Kane AA, Govier D, Marsh JL. Effect of distraction osteogenesis of the mandible on upper airway volume and resistance in children with micrognathia. Plast Reconstr Surg 2002: 109: 1809-1818.

29. Morovic, C. G., Monasterio, L. Distraction osteogenesis for obstructive apneas in patients with congenital craniofacial malformations. Plast. Reconstr. Surg. 105: 2324, 2000.

30. Cohen, S. R., Simms, C., Burstein, F. D. Mandibular distraction osteogenesis in the treatment of upper airway obstruction in children with craniofacial deformities. Plast. Reconstr. Surg. 101: 312, 1998.

31. Rodriguez, J. C., Dogliotti, P. Mandibular distraction in glossoptosis-micrognathic association: Preliminary report. J. Craniofac. Surg. 9: 127, 1998. 